\title{
Adverse effects of young maternal age on neonatal outcomes
}

\author{
Gavrielle Kang${ }^{1}$, MBBS, Jia Yi $\underline{\mathrm{Lim}}^{2}$, BSocSci, Anita Sugam Kale ${ }^{3}$, MBBS fRCOG, Le Ye $\underline{\operatorname{Lee}}^{4,5}$, MmBS, MRCPCH
}

INTRODUCTION Teenage pregnancy is associated with poor neonatal outcomes, which may burden the young mothers and their families. The aim of this study was to determine the effect young maternal age and single motherhood has on neonatal outcomes.

METHODS We conducted a retrospective cohort study of 267 infants born to mothers aged $\leq 21$ years in National University Hospital, Singapore, from January 2011 to December 2012. We compared the maternal demographics and neonatal outcomes of single mothers with those of married mothers. The neonatal outcomes of our study cohort were also compared to the hospital's birth cohort during the same period.

RESULTS Unsatisfactory antenatal care was more prevalent among the young single mothers than among the young married mothers (odds ratio [OR] 2.90, 95\% confidence interval [Cl] 1.71-4.92, $p<0.01$ ). The infants of the young single mothers had a lower mean birth weight $(p=0.01)$, with a significant proportion weighing $<2.5 \mathrm{~kg}(\mathrm{OR} 2.91,95 \% \mathrm{Cl} 1.35-6.37$, $\mathrm{p}<0.01$ ). Young maternal age was linked to a higher incidence of prematurity (OR $1.70,95 \% \mathrm{Cl} 1.18-2.43, \mathrm{p}<0.01$ ), major congenital defects (OR 4.68, 95\% Cl 2.10-10.13, $\mathrm{p}<0.01$ ), and a perinatal mortality of 18.7 per 1,000 births (OR 3.76, 95\% Cl 1.26-10.32, $\mathrm{p}=0.02$ ).

CONCLUSION Young single mothers were more likely to have unsatisfactory antenatal care and lighter infants. Young maternal age was associated with a higher risk of prematurity, major congenital malformations and perinatal mortality. More studies are needed to ascertain the cause of these adverse outcomes.

Keywords: antenatal care, neonatal mortality, prematurity, single motherhood, teenage pregnancy

\section{INTRODUCTION}

Teenage pregnancy is a significant public health issue, as it has been linked to an increased risk of obstetric complications and adverse neonatal outcomes. The findings of studies conducted worldwide over the past ten years on the cause of adverse outcomes in teenage pregnancy are contradictory. ${ }^{(1-12)}$ Some studies report that the adverse outcomes are due to physiological and anatomical factors associated with young maternal age, while others report that they are due to external factors such as socioeconomic status, social support, inadequate antenatal care and other behavioural determinants associated with adolescence. ${ }^{(1-12)}$ The adverse outcomes and complications linked to teenage pregnancy, such as the need for instrumental delivery or emergency Caesarean section, postpartum haemorrhage, prematurity, low birth weight, low Apgar score, neonatal intensive care unit (NICU) admission and perinatal mortality, ${ }^{(1-12)}$ are significant enough to warrant further studies to delineate the cause.

In Singapore, a higher incidence of low birth weight infants has been reported among young teenagers. ${ }^{(13)}$ Low birth weight, which is largely due to poor intrauterine growth, was found to result in a higher risk of low Apgar score at birth and a fivefold increased risk of perinatal mortality. ${ }^{(13)}$ However, that study did not evaluate the effect of maternal socioeconomic or behavioural factors on these outcomes. ${ }^{(13)}$ Based on data from the Singapore National Birth Defects Registry report in 2000, extreme maternal age was found to be a significant risk factor for non-chromosomal birth defects, a major cause of perinatal mortality locally. ${ }^{(14)}$ In particular, young mothers were found to have a higher risk of having a baby with gastroschisis, a congenital anterior abdominal wall defect. ${ }^{(15)}$ Although it has yet to be proven locally, gastroschisis has been shown to be linked to various socioeconomic and lifestyle factors (e.g. maternal cigarette smoking, alcohol use and recreational drug abuse) in studies conducted overseas. ${ }^{(16)}$ The two most common complications faced by young mothers locally are maternal anaemia and preterm labour; these complications are thought to be caused mainly by socioeconomic and psychosocial factors, such as unsatisfactory antenatal care and maternal nutrition, smoking, drug abuse and genital tract infections. ${ }^{(17)}$

As the prevalence of sexually active teenagers has remained steadily high over the last decade, ${ }^{(18-20)}$ the issue of teenage pregnancies and poor neonatal outcomes has increasingly wider implications on the social and economic situations in Singapore. Nonetheless, it is worth noting that the prevalence of sexually active teenagers does not translate to an equally high fertility rate in this age group. ${ }^{(21)}$

As young single mothers in Asian societies with traditional values tend to be viewed with stigma, they often do not receive the same financial and social benefits as married mothers. ${ }^{(22-24)}$ In Singapore, unmarried mothers were previously not entitled to the same duration of maternity leave as married mothers. They were eligible for only eight weeks of paid maternity leave (although some employers could have increased this to 12 weeks),

${ }^{1}$ Yong Loo Lin School of Medicine, National University of Singapore, ${ }^{2}$ Medical Social Work Department, ${ }^{3}$ Department of Obstetrics and Gynecology, ${ }^{4}$ Department of Neonatology, National University Hospital, ${ }^{5}$ Department of Pediatrics, Yong Loo Lin School of Medicine, National University of Singapore, Singapore

Correspondence: Dr Lee Le Ye, Consultant, Department of Neonatology, Level 12, NUHS Tower Block, 1E Kent Ridge Road, Singapore 119228. le_ye_LEE@nuhs.edu.sg 
a stark contrast to their married counterparts who were entitled to 16 weeks of paid maternity leave. However, this has been revised since 1 May 2013 to allow single mothers 12 weeks of paid maternity leave. ${ }^{(22)}$ Children born out of wedlock are also not eligible for cash benefits via the Baby Bonus Scheme. ${ }^{(22,23)}$ In addition, the typical profile of young single mothers (e.g. poor lifestyle habits, low educational qualifications, unemployment and lack of social support) ${ }^{(6,9)}$ predisposes them to be trapped in the vicious cycle of their social circumstance. Those in such circumstances can now approach the Community Development Council for assistance; some applicable assistance schemes from the Ministry of Family and Social Development are the ComCare Child Care Fees Assistance and ComCare Social Support Projects Fund. Unfortunately, most young single mothers are unaware of these available resources, even though they are arguably the demographic that needs the attention, resources and funding.

Further information on this vulnerable population would have the potential to affect community and social planning (e.g. in the area of educational intervention as well as the implementation and funding of targeted programmes). Therefore, the aim of the present study was to compare the health outcomes of neonates born to young single mothers in the local population to those of neonates born to young married mothers. This was done to affirm or dispute the associations of young maternal age and single motherhood with adverse outcomes, which have been established in other studies around the world.

\section{METHODS}

The present study was a retrospective cohort study of 267 infants who were born to mothers aged $\leq 21$ years in National University Hospital (NUH), Singapore, from January 2011 to December 2012. Ethics approval was obtained from the Domain Specific Review Board of the National Healthcare Group.

The effect of marital status on neonatal outcomes was studied by comparing the birth outcomes of infants born to single mothers to those of infants born to married mothers within our study population of mothers aged $\leq 21$ years. Mothers who were divorced $(n=1)$ were excluded from the analysis. The major neonatal morbidities of these 267 infants were also compared with the rest of the birth cohort (i.e. infants born to mothers aged $>21$ years who delivered in NUH during the study period, $n=5,542$ ). For the analysis of the effect of young maternal age on neonatal outcomes, the latter group of infants was used as a control population.

Data on the following variables were collected: (a) maternal age at delivery; (b) ethnicity; (c) marital status; (d) adequacy of antenatal care; and (e) lifestyle habits during pregnancy (e.g. smoking, alcohol consumption and drug abuse). The neonatal outcomes studied were: (a) gestational age at birth; (b) birth weight percentiles; (c) NICU admissions; (d) incidence of major congenital malformations; and (e) perinatal mortality (stillbirths and neonatal deaths in hospital up to day 28 of life). Information on the maternal demographics were extracted from the patients' admission documents. The demographic details of the mothers were routinely collected during the antenatal booking visit, upon admission to the obstetrics ward, and/or when the patient was reviewed by the medical social worker (MSW). Data on obstetric and neonatal outcomes were extracted from NUH's computerised clinical documents.

For mothers who had antenatal visits in the first trimester, the gestational age at birth was determined based on either the date of the mother's last menstrual period or the first trimester dating scans. For mothers who sought antenatal care after the first trimester, the gestational age of their infants was clinically determined using the Ballard scoring system. Infants were categorised as 'preterm' (i.e. < 32 weeks), 'late preterm' (i.e. 3236 weeks) or 'term' (i.e. $\geq 37$ weeks).

Antenatal care was classified as 'satisfactory' or 'unsatisfactory' based on how far along in the pregnancy the mothers were before they sought consultation for the first time and how regularly the mothers attended follow-ups. Mothers who had no visits booked, or who booked a visit after 16 weeks into the pregnancy (i.e. the window period for antenatal screening and potential interventions would have been missed) were classified as having unsatisfactory antenatal care. Antenatal care was also considered unsatisfactory if the number of follow-up visits was less than four, based on the World Health Organization (WHO)'s recommendation of a minimum of four antenatal visits. ${ }^{(25)}$

The birth weight centile of each infant was determined using the appropriate centile charts - the WHO's weight-for-age child growth standards for term girls and boys, ${ }^{(26)}$ and Fenton's fetal-infant growth chart for preterm infants. ${ }^{(27)}$ Low birth weight was defined as a birth weight of less than $2.5 \mathrm{~kg}$, and 'small for gestational age' was defined as a birth centile $\leq 10$ th percentile, based on the aforementioned centile charts, which take into account gestational age. Major congenital malformations were defined as structural defects present at the time of birth that required surgical intervention. Perinatal mortality included stillbirths and neonatal deaths occurring within the first 28 days of life.

Statistical analysis was performed using IBM SPSS Statistics software version 20.0 (IBM Corp, Armonk, NY, USA). Two-tailed Fisher's exact test was used to calculate the significance of the relative risk ratios, while independent samples $t$-test was used to compare the means. Mantel-Haenszel Chi-square test was used to determine whether there was any significant correlation between the variables and the respective outcomes. A p-value $<0.05$ was considered statistically significant.

\section{RESULTS}

The maternal characteristics of the study cohort are shown in Table I, while the neonatal outcomes are listed in Table II. Among the 267 young mothers in our study cohort, $69.7 \%$ were of Malay ethnicity and $50.2 \%$ were single mothers who had unplanned pregnancies. The median maternal age was 20 (range 15-21) years at delivery.

Antenatal care was unsatisfactory for $46.8 \%$ of the mothers, while $15.0 \%$ had absolutely no antenatal care (i.e. they presented only at delivery). Throughout the course of pregnancy, cigarette smoking was documented in $17.2 \%$ of the mothers, while alcohol consumption and substance abuse were documented in $2.6 \%$ and $0.7 \%$ of the mothers, respectively. 
Table I. Characteristics of the young mothers.

\begin{tabular}{|c|c|c|c|c|c|c|}
\hline \multirow[t]{2}{*}{ Characteristic } & \multicolumn{3}{|c|}{$\%$} & \multirow{2}{*}{$\begin{array}{l}\text { OR/mean } \\
\text { difference }\end{array}$} & \multirow[t]{2}{*}{$95 \% \mathrm{Cl}$} & \multirow[t]{2}{*}{ p-value } \\
\hline & All $(n=267)$ & Single $(n=134)$ & Married $(n=133)$ & & & \\
\hline \multicolumn{7}{|l|}{ Ethnicity } \\
\hline Chinese & 17.2 & 13.4 & 21.0 & 0.58 & $0.29-1.17$ & 0.11 \\
\hline Malay & 69.7 & 74.6 & 64.7 & 1.61 & $0.92-2.82$ & 0.09 \\
\hline Indian & 9.7 & 7.5 & 12.0 & 0.59 & $0.24-1.44$ & 0.22 \\
\hline Maternal age at delivery* (yr) & $20(15-21)$ & $19(15-21)$ & $20(15-21)$ & NA & NA & $<0.01$ \\
\hline \multicolumn{7}{|l|}{ Adequacy of antenatal care } \\
\hline Unsatisfactory & 46.8 & 59.7 & 33.8 & 2.90 & $1.71-4.92$ & $<0.01$ \\
\hline No antenatal care & 15.0 & 23.9 & 6.0 & 4.90 & $2.05-12.12$ & $<0.01$ \\
\hline \multicolumn{7}{|l|}{ Maternal behavioural factors } \\
\hline Cigarette smoking & 17.2 & 20.9 & 13.5 & 1.69 & $0.84-3.40$ & 0.14 \\
\hline Alcohol consumption & 2.6 & 3.7 & 1.5 & 2.54 & $0.43-19.26$ & 0.45 \\
\hline Substance abuse & 0.7 & 0.7 & 0.8 & 0.99 & $0.03-36.71$ & 1.00 \\
\hline
\end{tabular}

*Data presented as median (range). Cl: confidence interval; NA: not applicable; OR: odds ratio

Table II. Neonatal outcomes of infants born to young mothers.

\begin{tabular}{|c|c|c|c|c|c|c|}
\hline \multirow[t]{2}{*}{ Neonatal outcome } & \multicolumn{3}{|c|}{$\%$} & \multirow{2}{*}{$\begin{array}{l}\text { OR/mean } \\
\text { difference }\end{array}$} & \multirow[t]{2}{*}{$95 \% \mathrm{Cl}$} & \multirow[t]{2}{*}{ p-value } \\
\hline & All $(n=267)$ & Single $(n=134)$ & Married (n = 133) & & & \\
\hline Gender of baby* & 1.04 & 1.03 & 1.05 & 0.985 & $0.59-1.64$ & 1.00 \\
\hline Mean birth weight (g) & 2,876 & 2,795 & 2,959 & -164 & -292 to -37 & 0.01 \\
\hline Low birth weight $(<2.5 \mathrm{~kg})$ & 15.7 & 22.4 & 9.0 & 2.91 & $1.35-6.37$ & $<0.01$ \\
\hline Birth weight $\leq 10$ th percentile & 21.4 & 23.9 & 18.8 & 1.36 & $0.72-2.55$ & 0.37 \\
\hline \multicolumn{7}{|l|}{ Gestational age (wk) } \\
\hline$<32$ & 3.4 & 5.2 & 1.5 & 3.61 & $0.67-25.66$ & 0.17 \\
\hline $32-36^{+}$ & 11.6 & 11.2 & 12.0 & 0.92 & $0.41-2.07$ & 0.85 \\
\hline $37-42$ & 85.0 & 83.6 & 86.5 & 0.80 & $0.39-1.65$ & 0.61 \\
\hline NICU and special care admission & 10.5 & 14.2 & 6.8 & 2.28 & $0.93-5.70$ & 0.07 \\
\hline Major congenital defects & 3.4 & 3.7 & 3.0 & 1.25 & $0.28-5.69$ & 1.00 \\
\hline Incidence of stillbirths per 1,000 births & 7.5 & 7.5 & 7.7 & 0.99 & $0.03-36.71$ & 1.00 \\
\hline $\begin{array}{l}\text { Incidence of neonatal mortality per } \\
1,000 \text { livebirths }\end{array}$ & 11.2 & 14.9 & 7.5 & 2.00 & $0.14-56.42$ & 1.00 \\
\hline Emergency Caesarian section & 8.6 & 10.4 & 6.8 & 1.61 & $0.62-4.20$ & 0.38 \\
\hline
\end{tabular}

* Data presented as ratio of male-to-female babies. ${ }^{\text {Range }}=32$ weeks to 36 weeks and 6 days. Cl: confidence interval; NICU: neonatal intensive care unit; OR: odds ratio

The majority $(85.0 \%)$ of the infants were born at term, with only $3.4 \%$ born before 32 weeks. Major congenital defects (i.e. defects that require surgical intervention) were noted in $3.4 \%$ of the infants, and NICU and special care admissions were required for $10.5 \%$ of the infants. The incidence of stillbirths was 7.5 per 1,000 births, while the incidence of neonatal deaths was 11.2 per 1,000 livebirths. The distribution of birth weight percentiles in the study population was largely skewed toward the left of the curve (i.e. the infants were lighter). About $21 \%$ of the infants were small for gestational age (i.e. $\leq 10$ th percentile) and $79.0 \%$ were $\leq 50$ th percentile; $15.7 \%$ had low birth weight $(<2,500 \mathrm{~g})$.

Adjustment for maternal smoking during pregnancy and level of antenatal care showed no significant correlation between these variables and low birth weight $(p=0.91$ and $p=0.34$, respectively) and low birth centiles ( $p=0.35$ and $p=0.13$, respectively) (Table III). Although prematurity was found to be
Table III. Results of the Chi-square tests for potential confounders.

\begin{tabular}{llr}
\hline Variable & Outcome & p-value \\
\hline Maternal smoking & Prematurity & 0.53 \\
& Low birth weight $(<2.5 \mathrm{~kg})$ & 0.91 \\
& Small for gestational age & 0.23 \\
& Low birth centiles & 0.35 \\
Antenatal care & Prematurity & 0.34 \\
& Low birth weight $(<2.5 \mathrm{~kg})$ & 0.34 \\
& Small for gestational age & 0.14 \\
Prematurity & Low birth centiles & 0.13 \\
& NICU admissions & $<0.01$ \\
& Low birth weight $(<2.5 \mathrm{~kg})$ & $<0.01$ \\
& Small for gestational age & $<0.01$ \\
& Low birth centiles & 0.88 \\
\hline
\end{tabular}

Note: The Mantel-Haenszel Chi-square test was used to analyse for correlations between potential confounding variables and outcomes. All variable-outcome pairs with small cell counts were omitted; degrees of freedom $=1$. NICU: neonatal intensive care unit 
Table IV. Comparison of the outcomes of the study cohort with those of the rest of the birth cohort at the National University Hospital, Singapore, during the same period (January 2011-December 2012).

\begin{tabular}{lccccc}
\hline Outcome & \multicolumn{2}{c}{ \% } & OR & 95\% Cl & p-value \\
\cline { 2 - 4 } & Study cohort $(\mathbf{n = 2 6 7 )}$ & Control population* $(\mathbf{n}=\mathbf{5 , 5 4 2 )}$ & & \\
\hline Preterm births $(<37$ weeks) & 15.0 & 9.4 & 1.70 & $1.18-2.43$ & $<0.01$ \\
Major congenital defects & 3.4 & 0.7 & 4.68 & $2.10-10.13$ & $<0.01$ \\
Perinatal mortality per 1,000 births & 18.7 & 5.1 & 3.76 & $1.26-10.32$ & 0.02 \\
Stillbirths per 1,000 births & 7.5 & 3.1 & 2.45 & $0.39-11.10$ & 0.22 \\
Neonatal deaths per 1,000 livebirths & 11.2 & 2.0 & 5.71 & $1.26-22.17$ & 0.02 \\
NICU and special care admissions & 10.5 & 11.9 & 0.87 & $0.57-1.32$ & 0.56 \\
\hline
\end{tabular}

* Control population consists of the rest of the birth cohort in the National University Hospital, Singapore (i.e. infants born to mothers aged > 21 years). Cl: confidence interval; NICU: neonatal intensive care unit; OR: odds ratio

significantly associated with low birth weight $(p<0.01)$ and a birth centile $\leq 10$ th percentile (i.e. small for gestational age) ( $p<0.01)$, it did not account for the leftward skewing of the weight percentiles $(p=0.88)$. Among the young mothers, $61.0 \%$ required MSW referrals for reasons such as single motherhood, financial and/or social difficulties, troubled family circumstances and concerns regarding the care of their infants.

Tables I and II also include data on the young mothers and their infants, according to their marital status (i.e. single or married). The mean maternal age at delivery was found to be statistically lower for the single mothers than for the married mothers (19.03 years vs. 19.98 years, $p<0.01$ ). About $60 \%$ of the single mothers had unsatisfactory antenatal care, while only $33.8 \%$ of the married mothers had this problem (odds ratio [OR] 2.90, 95\% confidence interval [CI] 1.71-4.92, $\mathrm{p}<0.01$ ). In fact, $23.9 \%$ of the single mothers had never seen an obstetrician prior to delivery; this percentage was much lower among the married mothers (6.0\%) (OR 4.90, 95\% Cl $2.05-12.12, \mathrm{p}<0.01)$.

Infants born to single young mothers had a lower mean birth weight $(2,795 \mathrm{~g})$ than those born to married young mothers $(2,959 \mathrm{~g})(\mathrm{p}=0.01)$. The proportion of infants who were of low birth weight was 2.91 times higher among the single mothers when compared with the married mothers $(p<0.01)$. The incidence of early prematurity (i.e. gestational age $<32$ weeks) was also more common among the single mothers, although this difference was not statistically significant $(p=0.17$ ).

Overall, maternal marital status was not found to have any significant effect on gestational age, NICU admissions, major congenital abnormalities, mean Apgar scores (at both the first and fifth minute of life) and perinatal mortality. There was also no significant difference between the single and married mothers in terms of their maternal behavioural attributes (i.e. smoking, alcohol consumption and substance abuse during pregnancy), the incidence of obstetric complications, and the need for emergency operative delivery.

Table IV compares the outcomes of our study cohort ( $n=267$ ) with the rest of $\mathrm{NUH}^{\prime}$ s birth cohort during the same period (i.e. infants born to mothers aged $>21$ years, $n=5,542$ ). We found that with young maternal age, the OR for prematurity (i.e. gestational age $<37$ weeks) was 1.70 times higher $(95 \%$ Cl 1.18-2.43, $\mathrm{p}<0.01)$. Adjustment for possible confounders, such as maternal smoking and quality of antenatal care, showed no statistically significant correlation between these factors and prematurity. We also found that a significantly higher proportion of infants in the study cohort were born with major congenital defects as compared to the control population (3.4\% vs. $0.7 \%$; OR $4.68,95 \% \mathrm{Cl} 2.10-10.13, \mathrm{p}<0.01)$. If good antenatal care had been practiced, about $30 \%$ of these defects could have been detected before birth.

Death in the perinatal period was also significantly higher in our study cohort than in the control population (18.7 per 1,000 births vs. 5.1 per 1,000 births; OR 3.76, 95\% Cl 1.26$10.32, p=0.02)$. Specifically, neonatal mortality was found to be higher in the study cohort (11.2 per 1,000 livebirths) than in the control population (2.0 per 1,000 livebirths) (OR 5.71, 95\% Cl $1.26-22.17, p=0.02)$. These neonatal deaths were due to either extreme prematurity or lethal congenital malformation. Stillbirths, however, were not significantly higher in the study cohort (OR $2.45,95 \% \mathrm{Cl} 0.39-11.10, \mathrm{p}=0.22)$. Interestingly, the NICU and special care admissions of both groups were also not significantly different (OR 0.87, 95\% Cl 0.57-1.32, p=0.56).

\section{DISCUSSION}

The results of the present study show that young maternal age $(\leq 21$ years) is linked to a higher incidence of prematurity (OR 1.70), congenital malformations (OR 4.68) and neonatal death (OR 5.71). A distribution of birth weights that are predominantly around the lower percentiles was also observed among the young mothers. While the association between young maternal age and low birth weight is consistent with the conclusions of previous publications from Central Africa, ${ }^{(1)}$ Nepal, ${ }^{(3)}$ India, ${ }^{(5,12)}$ Nigeria ${ }^{(6)}$ the United States ${ }^{(8)}$ and Singapore, ${ }^{(13)}$ it contradicts the results of previous studies conducted in Nepal ${ }^{(7)}$ and Finland. ${ }^{(9)}$ These studies, however, used different cutoff ages to define 'young maternal age'. The following factors were found to contribute to the low birth weights of infants born to young mothers: (a) poor antenatal care; $^{(1,9)}$ (b) inadequate weight gain during pregnancy; ${ }^{(2)}$ and (c) maternal anaemia. ${ }^{(6)}$ Inadequate maternal weight gain was found to be a cause of low birth weight, prematurity and infant mortality in a study from Missouri, United States. ${ }^{(2)}$ Notably, younger mothers were found to have shorter and lighter statures, with body mass indexes that were lower than their older 
counterparts. ${ }^{(9,12)}$ However, the significance of this finding on infant birth weight is disputable.

Although young maternal age was associated with prematurity in studies conducted in Singapore, ${ }^{(17)}$ Nepal, ${ }^{(3,7)}$ India, ${ }^{(5,12)}$ Nigeria $^{(6)}$ and the United States, ${ }^{(8)}$ it was not found to have any significant effect on gestational age in publications from Central Africa ${ }^{(1)}$ and Finland. ${ }^{(9)}$ The following factors were found to contribute toward a higher incidence of prematurity among young mothers: (a) inadequate maternal weight gain; (2) (b) poor antenatal care; ${ }^{(5,17)}$ (c) low socioeconomic status; ${ }^{(6)}$ (d) maternal anaemia; ${ }^{(6,12,17)}$ and (e) negative lifestyle factors (e.g. smoking, alcohol consumption, drug abuse, poor nutrition and presence of genital tract infections). ${ }^{(17)}$

Pregnancy in adolescence was found to be a risk factor for neonatal mortality in studies conducted in India, ${ }^{(5)}$ Nigeria, ${ }^{(6)}$ the United States ${ }^{(8,10)}$ and Nepal, ${ }^{(11)}$ but not in studies conducted in Nepal, ${ }^{(3)}$ Brazil $^{(4)}$ and Finland..$^{(9)}$ The higher rates of neonatal mortality among young mothers could be explained by socioeconomic, ${ }^{(10)}$ behavioural and healthcare factors. ${ }^{(5,6)}$ Adjustment for these confounders showed that on its own, young maternal age was unlikely to be the cause of neonatal mortality. ${ }^{(5,10,11)}$ In essence, the findings of the present study are consistent with those of some studies, while contradicting others. A nationwide study on the pregnancy outcomes of young mothers will allow a more accurate picture of these factors.

We believe that the increased incidence of prematurity among young mothers is a potential contributing factor to the higher incidence of neonatal death observed in the same group. The higher incidence of severe congenital malformations in this group may also contribute to the higher incidence of neonatal deaths. When we compared the results of the present study to a local retrospective study of teenage pregnancy cases between 1994 and 2000, ${ }^{(14)}$ we found that the incidence of congenital defects was higher in our study cohort of young mothers. In both studies, only major congenital defects were considered. Some of these congenital defects and malformations could have been detected, with the potential for intervention, if antenatal obstetric care had been satisfactory. Unfortunately, about half of the young mothers in our study cohort had unsatisfactory obstetric care. The high perinatal and neonatal mortality rates in the present study are worrying (18.7 per 1,000 births and 11.2 per 1,000 livebirths, respectively), especially when compared to those of the general Singapore population (3.7 per 1,000 births and 1.2 per 1,000 livebirths, respectively). ${ }^{(28,29)}$

The lower birth weights observed among infants born to young mothers could have been due to a combination of factors, such as inadequate maternal nutrition, inadequate weight gain during pregnancy, ${ }^{(2)}$ shorter stature ${ }^{(13)}$ and anaemia during pregnancy; ${ }^{(6,9)}$ these factors were not investigated in the present study. A study by Kurth et al concluded that adolescent women had a significantly higher risk of having infants with lower birth weights; this was, in part, due to the lower frequency of antenatal visits among these women. ${ }^{(1)}$ Other studies with similar results failed to determine the exact causative factor that results in low birth weights among the infants of young mothers. ${ }^{(3,5,6,8)}$
As the proportion of young mothers who have infants with low birth weights is high, it is important that efforts to find the exact causative factor are not dampened.

In the present study, stratification of the data by adjusting for possible confounding variables showed that, on their own, lifestyle factors and the level of antenatal care were not causative in these adverse neonatal outcomes (i.e. low birth weight, prematurity, high incidence of congenital defects and high neonatal mortality). While a direct correlation between these outcomes and young maternal age cannot be proven, there is no doubt that an association exists. More studies will be needed to precisely determine the causative variable. It is likely that these outcomes are due to the physiological and anatomical traits of adolescent mothers, or a mix of several contributory factors, including behavioural and lifestyle choices, antenatal care and socioeconomic factors.

As the precise causative factors have yet to be identified, interventions that focus only on maternal lifestyle changes and antenatal health-seeking behaviour may not result in drastic improvements in neonatal outcomes. However, it is still beneficial to provide financial assistance and promote quality antenatal care, especially among young single mothers. ${ }^{(1,6,9)}$ In April 2013, Singapore's Ministry of Health revised the Medifund eligibility criteria such that it enabled support for needy patients requiring antenatal care. ${ }^{(30)}$ While such a revision can potentially benefit young single mothers, information on this Medifund revision needs to be actively disseminated to primary care physicians in order for optimal care and support to be achieved for this group of mothers. With better support, the detection of fetal abnormalities may be improved, allowing earlier interventions, and thus, lower incidences of neonatal morbidities.

Based on the results of the present study, it may be worthwhile to implement social measures targeted at vulnerable populations. These social measures should aim to lower the incidence of unplanned pregnancies in young and unmarried girls (e.g. through sex education and/or by promoting an awareness of the adverse neonatal outcomes associated with young maternal age). Young single mothers are especially vulnerable, as trends have shown that many of these mothers come from low-income families and have lower educational qualifications. As these mothers usually leave school early (at the primary or secondary level), they tend to have less employment opportunities. The high costs of raising a child superimposed with a lower earning capacity are likely to result in financial difficulties for these mothers. Knowledge of new policies ${ }^{(30)}$ implemented to help needy patients may encourage and improve support for basic antenatal care among single teenage mothers.

As the findings of the present study are based on data obtained from a single hospital (i.e. NUH, which is only one of the three restructured birthing hospitals in Singapore), they may not be representative of all teenage mothers in Singapore. Further studies are needed to uncover the incidence of teenage pregnancy in Singapore and the neonatal outcomes of this group of mothers. By gaining a better understanding of the situation, antenatal and neonatal support for this group can be improved. 
The present study was not without limitations. The low birth weights of the infants in our study cohort could have been due to a number of factors, including decreased placental nutrition (i.e. intrauterine growth restriction), prematurity, mothers who had smaller builds (due to their young age) and anomalies. Unfortunately, we were only able to study variables that were routinely documented in the case notes of obstetric patients. We were also not able to assess or adjust for other possible confounders for the associations studied, such as maternal nutrition, anaemia, stature, body mass index and adequacy of weight gained during pregnancy, due to poor follow-up in a significant proportion of the patients. This is a known inherent flaw of retrospective cohort studies. Also, although we found that preterm births could have been a possible confounding factor leading to infants with low birth weights and infants who were small for gestational age, prematurity did not account for the skewed distribution toward the lower birth weight percentiles.

In the present study, the number of neonatal morbidities was small. This meant that the stratification of outcomes by potential confounders could not be accurately analysed for all variables using the Mantel-Haenszel Chi-square test. Between the maternal groups (i.e. single vs. married), the numbers may not reach statistical significance due to the infrequent rates of neonatal morbidities in Singapore.

Trends in socioeconomic markers could only be studied in young mothers who were referred to MSWs. As these mothers were referred to MSWs precisely because of financial or social concerns, the generalisability of the demographic profile of these patients to all young single mothers in Singapore is limited. We were unable to accurately identify a specific population of adolescent girls who may be more vulnerable to single teenage motherhood, although a significant proportion of our study cohort was from low socioeconomic backgrounds, and had low levels of education and no employment. A population-based study should be conducted to obtain more accurate data regarding the effect of various socioeconomic markers on all young mothers in Singapore.

In terms of lifestyle factors during pregnancy, such as smoking, alcohol consumption and drug abuse, there is a significant potential for reporting bias. Our MSWs estimated that the actual numbers for maternal smoking are much higher than what was documented in the present study. In other words, maternal smoking could have been a silent confounding variable in our outcomes, as we had no way of adjusting for it.

Birth weight percentiles were charted based on WHO's weight-for-age child growth standards and Fenton's fetal-infant growth chart. These are international scales, which may not accurately represent the birth weight distribution of an Asian population. However, we were limited by the fact that Singapore does not have its own growth percentile charts. Furthermore, the birth weights in the control population were not studied, and therefore, could not be used as a direct measure for comparison.

Among the mothers who received adequate antenatal care (i.e. had opportunity for screening or diagnostic tests), there was the option of pregnancy termination in the event that severe fetal anomalies were detected antenatally. This could have resulted in a lower incidence of actual live births of infants with congenital malformations in the NUH dataset. However, this was only the case for mothers who had adequate antenatal care. The lack of satisfactory antenatal care in $46.8 \%$ of the young mothers in our study cohort could have accounted for the high rate of congenital malformations observed in the present study.

As NUH is a tertiary birthing unit, it receives many in utero transfers for infants with congenital malformations and complex cases requiring special or intensive neonatal care. Hence, a much higher baseline incidence of prematurity and major congenital malformations is present in our control population. This is reflected in the high special and intensive care admission rates in the baseline population.

To conclude, young maternal age is found to be associated with a higher risk of prematurity, congenital malformations, neonatal mortality and low birth weight percentiles. With the recent revision in the Medifund eligibility, which results in assistance being extended to this vulnerable group of mothers, we hope to see a reduction in the incidence of adverse neonatal outcomes. More studies need to be conducted to ascertain the exact causative variable that results in these outcomes.

\section{ACKNOWLEDGEMENTS}

We thank Eva Chow, Benjamin Png Ke Hao, Chua Wan Zhi, Christina d/o Anatharaju and Tang Kar Wai from the Department of Medical Social Work for their assistance in the care of the study cohort and in the formation of the database for the present study. We also thank Shireen Ong from the Obstetrics and Gynaecology Department for her assistance in retrieving the data needed, and Rajgor Dimple Dayaram for her assistance in editing, formatting and submitting the manuscript.

\section{REFERENCES}

1. Kurth F, Bélard S, Mombo-Ngoma G, et al. Adolescence as risk factor for adverse pregnancy outcome in Central Africa--a cross-sectional study. PLoS One 2010; 5:e14367.

2. Harper LM, Chang JJ, Macones GA. Adolescent pregnancy and gestational weight gain: do the Institute of Medicine recommendations apply? Am J Obstet Gynecol 2011; 205:140.e1-8.

3. Yadav S, Choudhary D, Narayan KC, et al. Adverse reproductive outcomes associated with teenage pregnancy. Mcgill J Med 2008; 11:141-4.

4. Restrepo-Méndez MC, Barros AJ, Santos IS, et al. Childbearing during adolescence and offspring mortality: findings from three population-based cohorts in southern Brazil. BMC Public Health 2011; 11:781.

5. Mukhopadhyay P, Chaudhuri RN, Paul B. Hospital-based perinatal outcomes and complications in teenage pregnancy in India. J Health Popul Nutr 2010; 28:494-500.

6. Ezegwui HU, Ikeako LC, Ogbuefi F. Obstetric outcome of teenage pregnancies at a tertiary hospital in Enugu, Nigeria. Niger J Clin Pract 2012; 15:147-50.

7. Stewart CP, Katz J, Khatry SK, et al. Preterm delivery but not intrauterine growth retardation is associated with young maternal age among primiparae in rural Nepal. Matern Child Nutr 2007; 3:174-85.

8. Chen XK, Wen SW, Fleming N, et al. Teenage pregnancy and adverse birth outcomes: a large population based retrospective cohort study. Int J Epidemiol 2007; 36:368-73.

9. Raatikainen K, Heiskanen N, Verkasalo PK, Heinonen S. Good outcome of teenage pregnancies in high-quality maternity care. Eur J Public Health 2006; 16:157-61.

10. Markovitz BP, Cook R, Flick LH, Leet TL. Socioeconomic factors and adolescent pregnancy outcomes: distinctions between neonatal and post- 
neonatal deaths? BMC Public Health 2005; 5:79.

11. Sharma V, Katz J, Mullany LC, et al. Young maternal age and the risk of neonatal mortality in rural Nepal. Arch Pediatr Adolesc Med 2008; 162:828-35.

12. Malviya MK, Bhardwaj VK, Chansoria M, Khare S. Anthropometric profile and perinatal outcome of babies born to young women ( $<18$ years). Indian Pediatr 2003; 40:971-6.

13. Kurup A, Viegas O, Singh K, Ratnam SS. Pregnancy outcome in unmarried teenage nulligravidae in Singapore. Int J Gynaecol Obstet 1989; 30:305-11.

14. Tan KH, Tan TY, Tan J, et al. Birth defects in Singapore: 1994-2000. Singapore Med J 2005; 46:545-52.

15. Tan KB, Tan KH, Chew SK, Yeo GS. Gastroschisis and omphalocele in Singapore: a ten-year series from 1993 to 2002. Singapore Med J 2008; 49:31-6.

16. Torfs CP, Velie EM, Oechsli FW, Bateson TF, Curry CJ. A population-based study of gastroschisis: demographic, pregnancy, and lifestyle risk factors. Teratology 1994; 50:44-53.

17. Nadarajah S, Leong NK. Adolescent pregnancies managed at KK Hospital. Singapore Med J 2000; 41:29-31.

18. YouthReach Resources. Statistics of Youth in Singapore (National Youth Council) [online]. Available at: http://www.youthreach.org.sg/resources/ youth-statistics.html. Accessed May 15, 2013.

19. Beyond Research. Child and Juvenile Statistics Singapore [online]. Available at: www.beyondresearch.sg/factsandstats.html\#17. Accessed May 19, 2013

20. Ng EH. Sexuality Education Programmes in Schools. 2009 Speech [online]. Available from http://www.moe.gov.sg/media/speeches/2009/05/21/ remarks-by-dr-ng-eng-hen-on-se.php. Accessed October 5, 2013.

21. Department of Statistics, Ministry of Trade \& Industry, Republic of Singapore. Yearbook of Statistics Singapore 2013 ISSN 0583-3655 [online]. Available at: http://www.singstat.gov.sg/publications/publications_and_ papers/reference/yearbook_2013/yos2013.pdf. Accessed July 15, 2013.

22. Section 9A, Child Development Co-Savings Act (Cap 38A, 2002 Rev Ed) [online]. Available at: http://www.mom.gov.sg/employment-practices/ leave-and-holidays/Pages/maternity-leave.aspx. Accessed October 8, 2013

23. Ministry of Social and Family Development. Baby Bonus Scheme [online]. Available at: http://www.babybonus.gov.sg/bbss/html/faq.html. Accessed July 15,2013

24. MT Wee. Social Security For Single-Parent Families In Singapore: Using A Social Investment Strategy To Solve The Moral Hazard Dilemma. In: Asian Journal of Public Affairs [online]. Available at: http://www.spp.nus. edu.sg/ajpa/pdf/issue1/Social_Security.pdf.Accessed July 14, 2013.

25. World Health Organization. Pregnancy, Childbirth, Postpartum, and Newborn Care: A guide for essential practice [online]. Available at: http:// www.who.int/maternal_child_adolescent/documents/924159084x/en/. Accessed March 3, 2015.

26. WHO Multicentre Growth Reference Study Group. WHO Child Growth Standards: Length/height-for-age, weight-for-age, weight-for-length, weight-for-height and body mass index-for-age: Methods and development. Geneva: World Health Organization 2006 [online]. Available at: http:// www.who.int/childgrowth/standards/technical_report/en/. Accessed March 3, 2015.

27. Fenton TR. A new growth chart for preterm babies: Babson and Benda's chart updated with recent data and a new format. BMC Pediatr 2003; 3:13.

28. Immigration and Checkpoints Authority of Singapore. Birth and Death Statistics. Singapore Demographic Bulletin 2012 [online]. Available at: http://www.ica.gov.sg/data/resources/docs/SDB_January\%202012.pdf. Accessed March 3, 2015.

29. Ministry of Health. Population and Vital Statistics [online]. Available at: http://www.moh.gov.sg/content/moh_web/home/statistics.html. Accessed July 13,2013

30. Gan KY. Better Health For All. Speech 2012 [onlinel. Available at: http:// www.moh.gov.sg/content/moh_web/home/pressRoom/speeches_d/2012/ moh_2012_committeeofsupplyspeechhealthcare2020improvingaccessibi. html. Accessed October 5, 2013. 